\title{
Influence of titanium precursor on photoluminescent emission of micro-cube-shaped $\mathrm{CaTiO}_{3}$
}

\author{
Tatiana Martelli Mazzo a,*, Gabriela Santilli do Nascimento Libanori ${ }^{\text {a }}$, \\ Mario Lucio Moreira ${ }^{\mathrm{b}}$, Waldir Avansi Jr. ${ }^{\mathrm{c}}$, Valmor Roberto Mastelaro ${ }^{\mathrm{d}}$, José Arana Varela ${ }^{\mathrm{e}}$, \\ Elson Longo ${ }^{\mathrm{e}}$ \\ a Departamento de Ciências do Mar, Universidade Federal de São Paulo, Avenida Almameda Saldanha da Gama, 89, Ponta da Praia, CEP 11030-400 Santos, SP, \\ Brazil

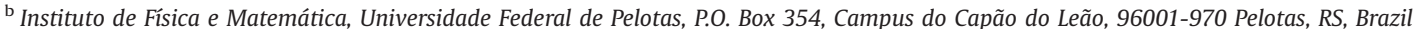 \\ c Departamento de Física, Universidade Federal de São Carlos, Jardim Guanabara, 13565-905 São Carlos, SP, Brazil \\ d Instituto de Física de São Carlos, Universidade de São Paulo, Av. Trabalhador São Carlense, 400, Arnold Schimidt, 13566-590 São Carlos, SP, Brazil \\ e INCTMN/LIEC, Instituto de Química, Universidade Estadual Paulista, P.O. Box 355, R. Francisco Degni, 55, Bairro Quitandinha, 14801-907 Araraquara, SP, \\ Brazil
}

\section{A R T I C L E I N F O}

\section{Article history:}

Received 5 November 2014

Received in revised form

10 March 2015

Accepted 23 March 2015

Available online 17 April 2015

\section{Keywords:}

Photoluminescencence

$\mathrm{CaTiO}_{3}$

Microwave-assisted hydrothermal

\begin{abstract}
A B S T R A C T
For this research, we studied the influence of titanium tetrachloride (TC) and titanium tetraisopropoxide (TTP) precursors on $\mathrm{CaTiO}_{3}$ (CTO) synthesis by employing a microwave-assisted hydrothermal (MAH) method regarding their respective short-, medium- and long-range features to determine if the use of different titanium precursors enhances the structural evolution of the material. The growth mechanism for the formation of the micro-cube-shaped CTO is proposed to obtain nanoparticle aggregation of selfassembly nanoplates. The disorder coupled to the oxygen vacancies of $\left[\mathrm{TiO}_{5}\right]-\left[\mathrm{TiO}_{6}\right]$ in complex clusters in the CTO 1 powder and twists in bonding between the $\left[\mathrm{TiO}_{6}\right]-\left[\mathrm{TiO}_{6}\right]$ complex clusters in the CTO 2 powder were mainly responsible for photoluminescent $(\mathrm{PL})$ emission.
\end{abstract}

(c) 2015 Elsevier B.V. All rights reserved.

\section{Introduction}

Optical properties of perovskites (particularly luminescent properties) have attracted considerable attention from many researchers [1-3]. CTO powders possess important PL properties at room temperature and can be applied as dielectric resonators in wireless communication systems [4]. Previously published research reported on luminescent CTO powders which were obtained by the MAH method at $140{ }^{\circ} \mathrm{C}$ for $10 \mathrm{~min}$ [5]. The MAH method was developed by a number of researchers [5-7] and is considered as a genuine low temperature procedure [8] which utilizes high heating rates [9] and short annealing times where the interaction of microwave radiation with matter is observed.

X-ray absorption near edge spectroscopy (XANES) has been used to obtain information about the coordination environment of metal in oxide materials since the XANES region is highly sensitive to disorder short and medium ranges around the metal atom. For instance, Ti K-edge XANES experimental results for PL titanates revealed the coexistence of two types of environments for

\footnotetext{
* Corresponding author. Tel.: +55 1335235063.

E-mail address: tatimazzo@gmail.com (T.M. Mazzo).
}

titanium before reaching the complete structural order: five-fold coordination $\left(\mathrm{TiO}_{5}\right.$, square-base pyramid) and six-fold coordination $\left(\mathrm{TiO}_{6}\right.$, octahedron) [10-12].

$\mathrm{PL}$ is a powerful probe for certain aspects of the medium-range order structure of 5-20 A; e.g., clusters where the degree of local order is such that structurally inequitable sites can be distinguished because they have different types of electronic transitions and are linked to a specific structural arrangement. PL emission in perovskite materials is considered to be closely related to crystal structures and their corresponding distorted metaloxygen polyhedra; this disorder has been attributed to the presence of different coordination modes in titanium $[5,13,14]$.

Several studies reported PL properties of titanate compounds which were related to the structural disorder of the titanium environment $[5,7,13,14]$. For example, quasi-amorphous structures obtained by the polymeric precursor method (Pechini) were associated with the presence of distorted $\left[\mathrm{TiO}_{6}\right]-\left[\mathrm{TiO}_{5}\right]$ complex clusters which are responsible for luminescent emission [13]. These PL properties are probably related to the level of structural organization, preparation method and thermal treatment conditions. Thus, innovative and profitable processing methods are 
proposed to investigate several levels of structural organization which are linked to PL emission at room temperature.

In this study, CTO powders were successfully prepared by the MAH method using TC and TTP as titanium precursors at $140{ }^{\circ} \mathrm{C}$ for $4 \mathrm{~min}$. Despite its excellent results, TC is an expensive reagent which is highly reactive and dangerous $[5,8,15]$. Therefore, TTP was substituted since it is an inexpensive and easily manipulated reagent. The main objective is to investigate the impact of precursors on the former network for structural order-disorder and PL emission associated with $\mathrm{TiO}_{6}$ octahedral cluster behavior of CTO powders prepared by the MAH method.

\section{Experimental section}

The experimental procedure was conducted using two different titanium (Ti) precursors: TC and TTP. First, $0.01 \mathrm{~mol}$ of the Ti precursor was slowly added to $30 \mathrm{~mL}$ of deionized water at $0{ }^{\circ} \mathrm{C}$. Each solution of TC and TTP was mixed with $0.01 \mathrm{~mol}$ of $\mathrm{CaCl}_{2} .2 \mathrm{H}_{2} \mathrm{O}$ singly and then mixed under constant stirring. Finally, $50 \mathrm{~mL}$ of $\mathrm{KOH}$ solution $(6.0 \mathrm{M})$ was added as a precipitation agent. The two solutions were identified as CTO 1 using TTP precursor and CTO 2 using TC precursor. Each solution was loaded into a $110 \mathrm{~mL}$ Teflon autoclave separately; the autoclave was sealed and placed in the MAH system at $140{ }^{\circ} \mathrm{C}$ for $4 \mathrm{~min}$. The solid products were washed with de-ionized water until a neutral $\mathrm{pH}$ and then dried at $60^{\circ} \mathrm{C}$ for $12 \mathrm{~h}$.

CTO 1 and CTO 2 powders were characterized by X-ray diffraction (XRD) in a Rigaku Dmax 2500PC diffractometer using $\mathrm{Cu}$ $\mathrm{K} \alpha(1=1.5406 \AA)$ radiation. Data were collected from $20^{\circ}$ to $70^{\circ}$ in the $2 \theta$ range with a $0.5^{\circ}$ divergence slit and a $0.3 \mathrm{~mm}$ receiving slit using a fixed-time mode with a $0.02^{\circ}$ step size and a $1 \mathrm{~s} /$ point rate. Raman spectroscopy data were obtained at room temperature using RFS/100/S Bruker FT-Raman equipment with a spectral resolution of $4 \mathrm{~cm}^{-1}$ attached to a Nd:YAG laser which produced an excitation light of $1.064 \mathrm{~nm}$ in the frequency range of 100 up to $1000 \mathrm{~cm}^{-1}$. Ultraviolet-visible (UV-vis) absorption spectra of CTO powders were taken using a total diffuse reflectance mode in Cary $5 G$ equipment. PL data for the powders were obtained using a Jobin Yvon-Fluorolog III spectrofluorometer at room temperature under the excitation of a $450 \mathrm{~W}$ xenon lamp.

The local atomic structure around titanium (Ti) atoms was studied by X-ray absorption spectroscopy (XAS). XANES spectra were measured at the titanium Ti K-edge using a D04B-XAFS1 beam line at the Brazilian Synchrotron Light Laboratory (LNLS). Ti K-edge XANES spectra were collected on samples deposited on polymeric membranes in transmission mode at room temperature with a Si (1 111 ) channel-cut monochromator. Spectra were measured from $30 \mathrm{eV}$ below and $150 \mathrm{eV}$ above the edge with an energy step of $0.3 \mathrm{eV}$ near the edge region. To provide better energy reproducibility during spectra collection, the energy calibration of the monochromator was checked during data collection using a Ti metal foil. Finally, microstructural characterization was performed by field emission scanning electron microscopy (FE-SEM) using Zeiss Supra ${ }^{T M} 35$ equipment.

\section{Results and discussion}

The polycrystalline nature of CTO powders is illustrated by XRD patterns depicted in Fig 1 and were identified by JCPDS card (no. 420423) as an orthorhombic phase showing the Pbnm space group.

The $\mathrm{CaCO}_{3}$ phase is visible in the CTO 1 powder and is identified by JCPDS card (no. 5-543). CTO 1 and CTO 2 powders revealed a crystalline profile which indicates that structurally long-range ordered powders are obtainable at this temperature. An analysis (see Fig. 1) identifies the formation of calcium titanate prepared by the MAH method using two different Ti precursors at low temperatures and

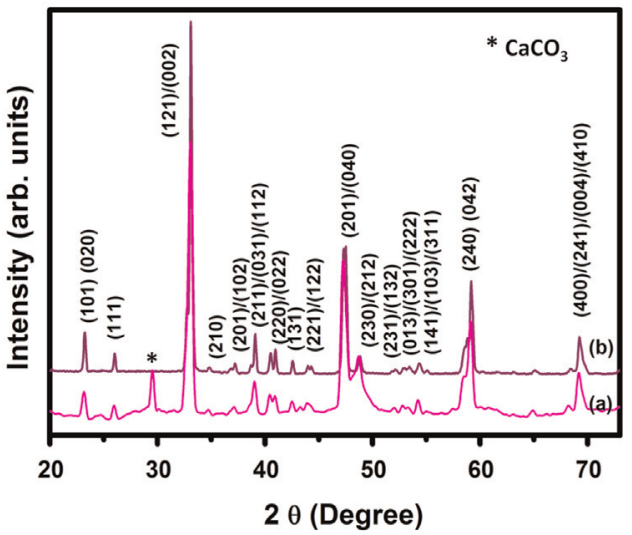

Fig. 1. XRD patterns for CTO powders synthesized at $140{ }^{\circ} \mathrm{C}$ for 4 min with (a) CTO 1 and (b) CTO 2.

very short reaction times. The fast reaction is attributed to the microwave coupling of water bonds for a hydroxide complex cluster in an aqueous sheath which promotes uniform heating of the whole solution $[16,17]$. СTO micro-cubes were obtained using shorter times as compared with our previous research [5]. The crystalline degree of CTO 2 is apparently better than the crystalline degree of CTO 1; moreover, it has no spurious phases. Fig. 2 shows micro-cube CTO produced via the self-organization of nanoplates which possess a similar morphology of calcite crystals [18]. This mechanism is known as "self-assembly"; the interaction between pre-defined smaller individuals of the same origin results in a specific form which is highly ordered and ends spontaneously.

Examples of nanoplates exhibiting a regular external polyhedral morphology are still relatively rare [19], and the detailed mechanisms are still largely not understood. As a general rule, kinetic polymorph control for inorganic materials is based predominantly on the modification of the activation-energy barriers, nucleation, growth of particle over particles like assembles [20,21], and phase transformation [22]. In these cases, the crystallization is often a sequential step involving structural and compositional modifications rather than a multi-step pathway [23-25].

A nucleation mechanism for the formation of micro-cube-shaped CTO in terms of chemical reactions and crystal growth is described as follows (see Fig. 2): the clusters are considered as constituent polyhedra of the perovskite based materials, i.e. $\mathrm{TiO}_{6}$ (octahedral clusters) and $\mathrm{CaO}_{12}$ (dodecahedral clusters). To close this discussion, it is important to consider the structural organization that occurs in the synthesis process. In solution, titanium and calcium ions are chelated by $\mathrm{H}_{2} \mathrm{O}$ and $\mathrm{OH}$ groups, and an almost regular $\mathrm{TiO}(\mathrm{OH})_{2} \cdot x \mathrm{H}_{2} \mathrm{O}$ system without constraints is therefore formed which is surrounded by a random distribution of $\mathrm{Ca}(\mathrm{OH})_{2}$. At this point, a certain kind of organization already exists at short range as a polarized structure. After placing the reaction solution in the MAH system, the cluster dehydration begins to set in a rigid $2 \mathrm{D}$ or $3 \mathrm{D}$ network. This process is controlled by the $\mathrm{OH}$ and $\mathrm{H}_{2} \mathrm{O}$ loss of $\mathrm{TiO}(\mathrm{OH})_{2} \cdot x \mathrm{H}_{2} \mathrm{O}$ and $\mathrm{Ca}(\mathrm{OH})_{2}$ complex hydrated clusters. Then a quasi-crystal polarized phase originates from a polarized phase where the dehydrated clusters start to generate a periodic organization which characterizes a typical transition phase from hydrated clusters to first Fig. 2 [26]. The complete crystallization is achieved when a long-range organization appears (see Fig. 3) in addition to local order which sometimes deviates from the ideal structure due to constraints induced by the long-range ordering. The next step is the formation of nanoplates, and the sequence is completed by the self-assembly of nanoplates via hydrogen bonds formed among $\mathrm{OH}$ surface groups to yield microcube-shaped CTO; a schematic representation of these stages is displayed in Fig. 2. In the second step, the agglomeration process occurs 
which can happen in an appropriate intermediate interaction region. The colloidal stabilization of the nanoparticles must be so weak that two nanoparticles can approach each other within the primary minimum. This minimum is achieved but the flexibility and dynamics must still be high enough to rearrange to the low energy configuration represented by a coherent particle-particle interface [27]. Our main candidates to facilitate the coherent interaction between CTO nanoplates are the hydrogen bonds between $\mathrm{OH}$ groups which avoids the coalescent process at short times and originates a self-assemble organization (see Fig. 2).

According to the group theory calculations [28-30], a $\mathrm{CaTiO}_{3}$ with a perovskite-type orthorhombic structure and a space group of Pbnm with four clusters per unit cell exhibit 24 Raman-active modes which are represented by Eq. (1):

$\Gamma($ raman $)=7 A_{g}+5 B_{1 g}+7 B_{2 g}+5 B_{3 g}$

Raman-active modes in the FT Raman spectrum are produced from the stretching, torsional and bending vibrations of the metal oxygen bonds in the CTO perovskite-type crystalline structure.

However, small changes in polarizability in the orthorhombic CTO lattice [31] caused by the MH method can improve the interaction between the ions and also promote the overlapping of Raman active modes with modes of low intensity. Thus, only nine Raman active
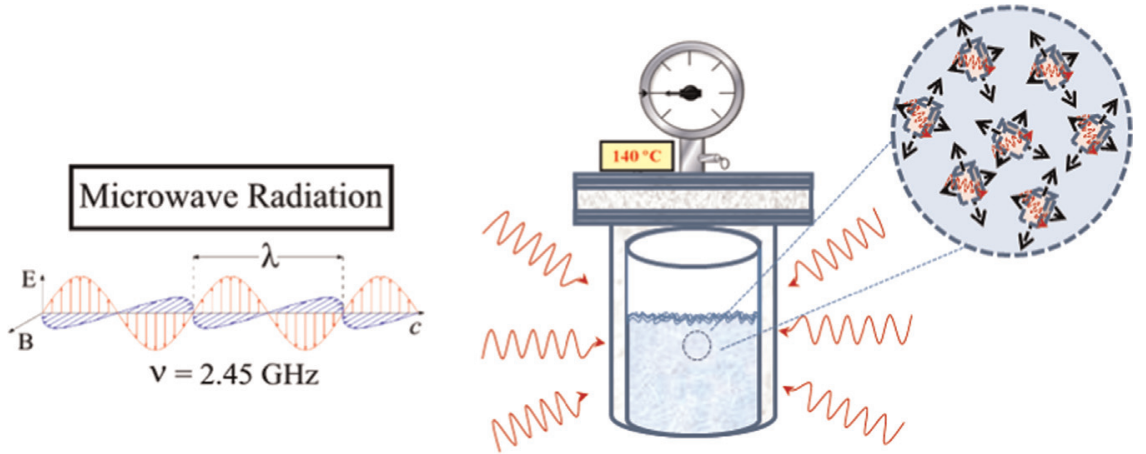

3D Periodic Lattice Formation

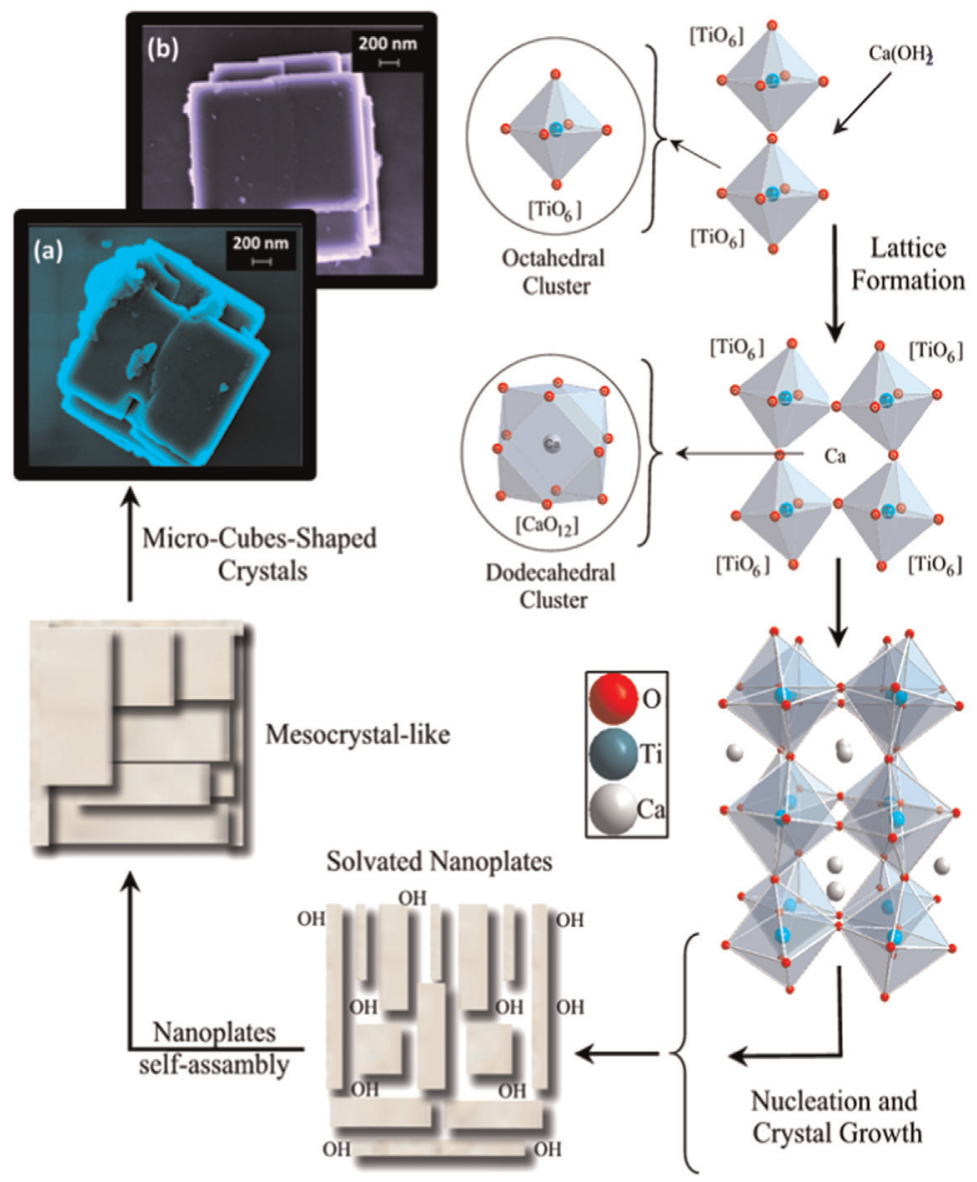

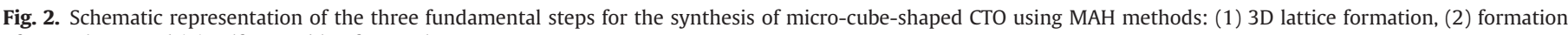
of nanoplates, and (3) self-assembly of nanoplates. 

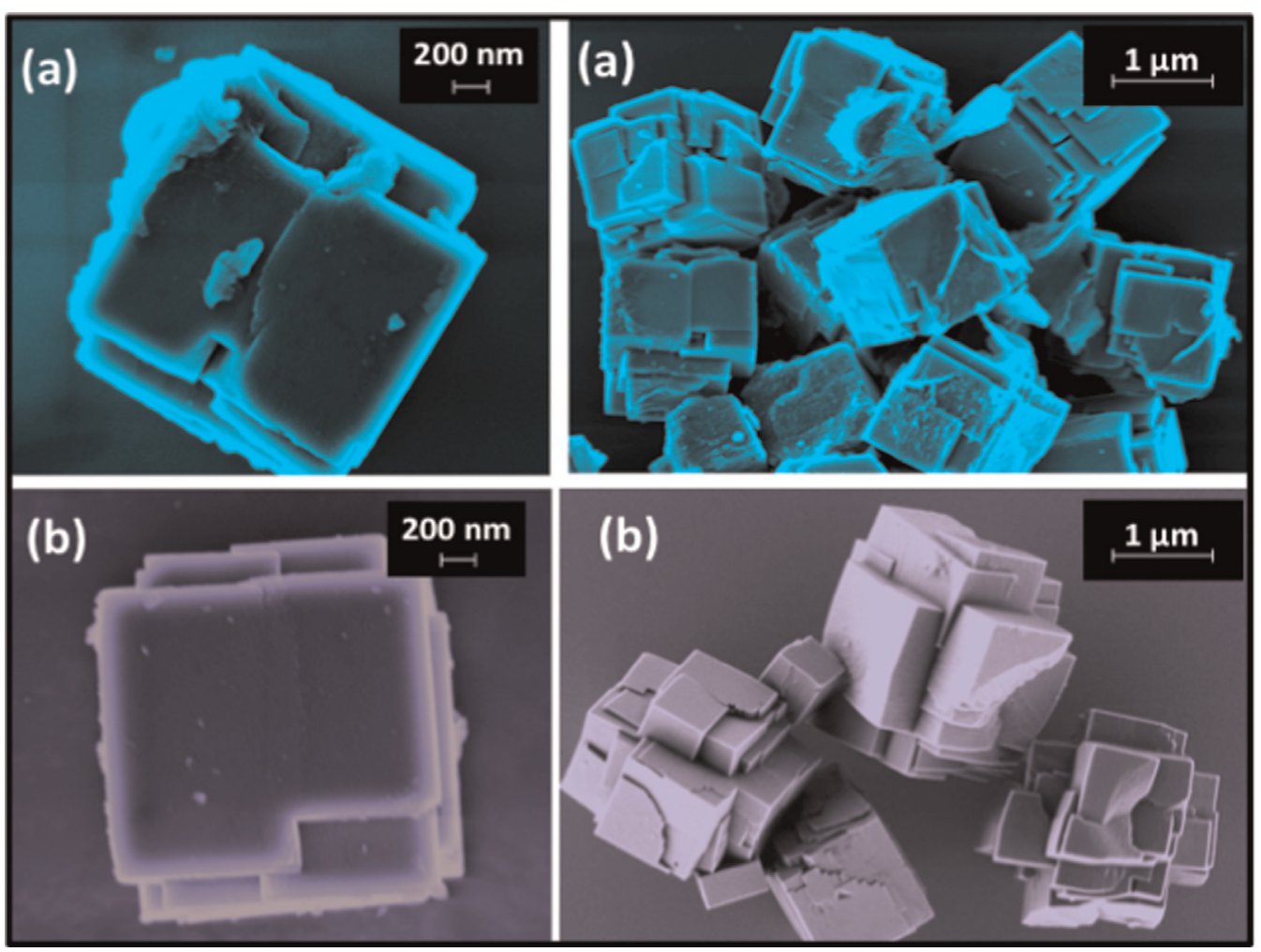

Fig. 3. FE-SEM micrograph of (a) CTO 1 and (b) CTO 2 powders.

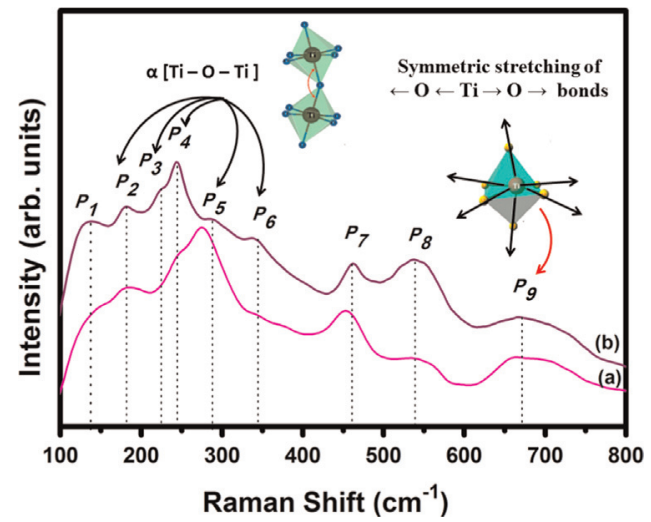

Fig. 4. FT-Raman spectra for (a) CTO 1 and (b) CTO 2 powders.

modes $\left(\mathrm{P}_{1}, \mathrm{P}_{2}, \mathrm{P}_{3}, \mathrm{P}_{4}, \mathrm{P}_{5}, \mathrm{P}_{6}, \mathrm{P}_{7}, \mathrm{P}_{8}\right.$ and $\left.\mathrm{P}_{9}\right)$ were detected from the 24 Raman-active modes in FT Raman spectra of CTO 1 and CTO 2 powders. Characteristic FT-Raman spectra of CTO 1 and CTO 2 powders depicted in Fig. 4 indicate good organization in short-range approaches to CTO 1. Spectra reveal nine vibrational modes which are in agreement with previous reports on CTO compounds [5,17,32,33]. Raman active modes are commonly observed: $134 \mathrm{~cm}^{-1}\left(\mathrm{P}_{1}\right)$ for a vibration of $\mathrm{Ca}$ bonded to a $\mathrm{TiO}_{3}$ group $\left(\mathrm{Ca}-\mathrm{TiO}_{3}\right)$ lattice mode; 181 , $224,244,287$ and $339 \mathrm{~cm}^{-1}\left(\mathrm{P}_{2}, \mathrm{P}_{3}, \mathrm{P}_{4}, \mathrm{P}_{5}\right.$ and $\left.\mathrm{P}_{6}\right)$ are associated with $\mathrm{O}-\mathrm{Ti}-\mathrm{O}$ bending modes or caused by the tilting phenomenon between $\left[\mathrm{TiO}_{6}\right]-\underline{\alpha}-\left[\mathrm{TiO}_{6}\right]$ clusters [34] (see the inset of Fig. 2). 464 and $495 \mathrm{~cm}^{-1}\left(\mathrm{P}_{7}\right.$ and $\left.\mathrm{P}_{8}\right)$ Raman peaks are attributed to torsional modes while the $\mathrm{P}_{9}$ peak is assigned to the Ti-O symmetric stretching vibration mode [35-38]. Shifts observed on the characteristic positions of Raman peaks of the СТО 1 as compared to the pure СТО 2 powder can be related to the symmetry breaking of $\left[\mathrm{TiO}_{6}\right]$ clusters. This effect produces structural defects such as distortions and/or strains in the crystal structure that can modify the rotational modes of the $-\mathrm{O}-\mathrm{Ti}-\mathrm{O}-$ bonds in octahedral $\left[\mathrm{TiO}_{6}\right]$ clusters. As shown by
Table 1

Data obtained by Raman spectra analyses of CTO in comparison with other research reported in the literature.

\begin{tabular}{lllllll}
\hline Vibrational modes & & Ref. [5] & Ref. [36] & Ref. [39] & CTO 1 & CTO 2 \\
\hline $\begin{array}{l}\text { (Ca-TiO) } \\
\text { Lattice mode }\end{array}$ & $\boldsymbol{P}_{\mathbf{1}}$ & 134 & 155 & 155 & 134 & 134 \\
(O-Ti-O) & & & & & & \\
Bending modes & $\boldsymbol{P}_{\mathbf{2}}$ & 181 & 180 & 184 & 181 & 181 \\
& $\boldsymbol{P}_{\mathbf{3}}$ & 224 & 226 & 228 & 223 & 223 \\
& $\boldsymbol{P}_{\mathbf{4}}$ & 244 & 247 & 249 & 247 & 244 \\
& $\boldsymbol{P}_{\mathbf{5}}$ & 287 & 286 & 287 & 275 & 284 \\
& $\boldsymbol{P}_{\mathbf{6}}$ & 339 & 337 & 340 & 340 & 339 \\
(Ti-O $)$ & $\boldsymbol{P}_{\mathbf{7}}$ & 464 & 471 & 471 & 460 & 453 \\
Torsional modes & $\boldsymbol{P}_{\mathbf{s}}$ & 537 & 495 & 497 & 539 & 539 \\
(Ti-O) & $\boldsymbol{P}_{\mathbf{9}}$ & 669 & 639 & 637 & 669 & 669 \\
Stretching modes & & & & & & \\
\hline
\end{tabular}

Cavalcante et al. [39], peak positions in the Raman spectrum have a small shift according to the method used to prepare the compounds. In Raman spectra, the ordering-disordering scheme may affect the number, width and shape of peaks. The shift and intensity of specific bands in the high-frequency range are expected to be associated with the vibration of distorted octahedra [38]. Table 1 illustrates that Raman positions in this study are close to positions reported in the literature; small deviations are influenced by the MAH method employed and distortions and defects into lattice due to different aspects of $\mathrm{Ti}$ precursors utilized. These results demonstrate that when TC is used, the structure formed is more ordered in the shortrange as reported by XRD results (see Fig. 1). When using TC as a titanium precursor, the lattice formation is almost instantaneous because $\mathrm{Ti}^{4+}$ ions are available in the solution (Eq. 2) due to its ionic character. On the other hand, when using TTP, the lattice formation may be slower due to its more covalent character; hence its solubility in water is lower as compared to TC. For TTP, the formation of the $\mathrm{TiO}(\mathrm{OH})_{2}$ precipitates occurs in two steps (Eqs. (3) and (4)). Moreover, the hydrolysis of TTP produces $\mathrm{C}_{3} \mathrm{H}_{7} \mathrm{OH}$ as a reaction product (Eq. (4)) which has a lower dielectric constant than water. The 

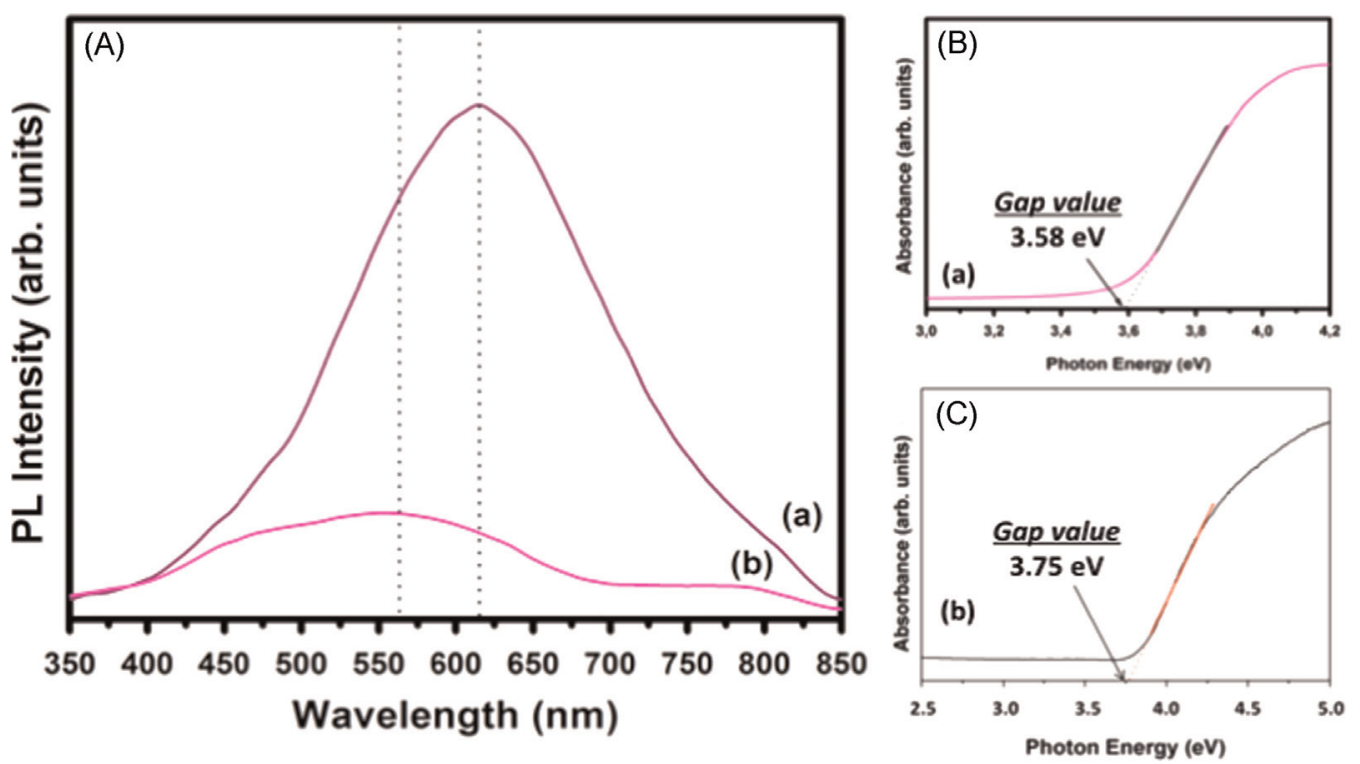

Fig. 5. (A) PL spectra and (B and C) absorption spectra of (a) CTO 1 and (b) CTO 2 powders.

magnitude of microwave warming depends on dielectric properties of substances. Therefore, $\mathrm{C}_{3} \mathrm{H}_{7} \mathrm{OH}$ in the CTO 1 reaction decreases the dielectric constant which may be a disadvantage to microwave heating, and therefore the material obtained is more disordered in the long and short range.

$\mathrm{TiCl}_{4(\mathrm{l})}+4 \mathrm{OH}^{-}{ }_{(\mathrm{aq})}^{\stackrel{\mathrm{KOH}}{\Longrightarrow}} \mathrm{Ti}(\mathrm{OH})_{4(\mathrm{~s})}+4 \mathrm{Cl}^{-}{ }_{(\mathrm{aq})}$

$\left[\mathrm{TiOCH}\left(\mathrm{CH}_{3}\right)_{2}\right]_{4(\mathrm{l})}+2 \mathrm{H}_{2} \mathrm{O} \stackrel{\mathrm{KOH}}{\Longrightarrow} \mathrm{TiO}_{2(\mathrm{~s})}+4\left(\mathrm{CH}_{3}\right)_{2} \mathrm{CHOH}_{(\mathrm{l})}$

$\mathrm{TiO}_{2(\mathrm{~s})}+4 \mathrm{OH}^{-}{ }_{(\mathrm{aq})} \stackrel{\mathrm{KOH}}{\Longrightarrow} \mathrm{TiO}(\mathrm{OH})_{2(\mathrm{~s})}+\mathrm{H}_{2} \mathrm{O}_{(\mathrm{l})}$

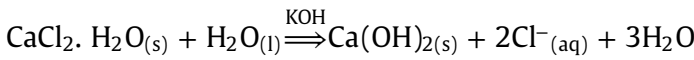

These structural features can also be observed on optical gaps (see Fig. 5B and C) obtained by extrapolation of linear curve regions according to the Wood and Tauc method [40], and they are $\mathrm{eV}$ for the CTO 2 powder and 3.58 for the CTO 1 powder, respectively; absorption are shown in Fig. 5. These results indicate that the exponential optical absorption edge and the optical bandgap energy are controlled by the degree of structural disorder in the lattice. When TTP (CTO 1 powder) was used, a decrease in the band-gap of structurally ordered powder can be attributed to defects and/or local bond distortion which provoke a redistribution in the density of states and an introduction of intermediate electronic defect states in the band gap [11] supports FT-Raman results.

The PL emission behavior of materials obtained by both CTO 1 and CTO 2 powders and reference compounds (CTO 400 and CTO 600 ) with a wavelength of $350.7 \mathrm{~nm}$ excitation shows a broad band spectrum at room temperature in the range of $350-580 \mathrm{~nm}$. Curves of PL CTO powders synthesized are shown in Fig. 6. These curves illustrates a more defined peak at $554 \mathrm{~nm}$ in the CTO 2 powder and a second emission component preceded by the issue of a broad band. This secondary emission peak is related to low energy transitions, and its emission maximum is located at $778 \mathrm{~nm}(1.6 \mathrm{eV})$. In the PL emission of the CTO 1 powder, the maximum emission is centered at $615 \mathrm{~nm}$. For the CTO 400 and 600 the the maximum emission is centered at 605 and $519 \mathrm{~nm}$, repectively. Emissions were observed for the all CTO powders range from ultraviolet to infrared in a composition of bands and peaks with varying contributions in each region of the spectrum. This result was expected since the absorption of light (according to the UV-vis absorbance spectra) suggests much lower energies than the CTO band gap. Absorption spectra verify that the former CTO 1 and CTO 2 absorptions occur even below $2.6 \mathrm{eV}$.

Emission spectra of CTO 1, CTO 2, and a reference, CTO compound synthesized by the Pechini method and heat-treated at $600{ }^{\circ} \mathrm{C}$ (CTO 600) and $400{ }^{\circ} \mathrm{C}$ (CTO 400), respectively, were decomposed using a Gaussian function (see Fig. 6). This decomposition was accomplished using the PeakFit program (version 4.05). The Gaussian function was used successfully to fit the PL peaks due to its great consistance with original PL spectra and tuning parameters, including the position of the peaks, and the corresponding percentage areas are recorded in Table 2.

The decomposition can be interpreted as a composition of five components that as as follows: a violet component (maximum below $438 \mathrm{~nm}$ ); a blue component (maximum intensity of $\mathrm{PL}$ around $500 \mathrm{~nm}$ ); a green component (maximum intensity of PL around $590 \mathrm{~nm}$ ); a red component (maximum intensity of PL less than $740 \mathrm{~nm}$ ); and a component which extends to an allusion in the near infrared region where the maximum intensity of each component appears as PL precursors as a lattice former.

Analyzing the values of percentage area for the CTO 2 powder, we observed that the maximum contribution is in the blue and green light range; for the CTO 1 powder, the maximum contribution is in the red light range; for the CTO 400 powder, the maximum contribution is in the red light range and for the CTO 600 powder, the maximum contribution is in the green light range. The violet and blue emission can be attributed to a more ordered structure while the green, yellow and red emissions are linked to a greater disorder in the lattice [11]. With these results we can conclude that the СTO 1 and CTO 600 samples have a higher structural organization. The Commission International d'Eclairage (CIE) chromaticity coordinates is a tool to obtain information about brightness and efficiency of luminescence [41]. The values for the samples are CTO $1(x=0.537 y=0.380)$, СТО 2 $(x=0.392 y=0.372)$, СТО $400(x=0.510 y=0.384)$ and СТО 600 $(x=0.372 y=0.383)$. The CTO 1 and CTO 400 samples being values localized in the red emission region, the CTO 2 and CTO 600 being values localized in the yellow and green region, respectively, as illustrated in Fig. 7. This results corroborate with the PL decomposition spectra emissions (see Fig. 6). 

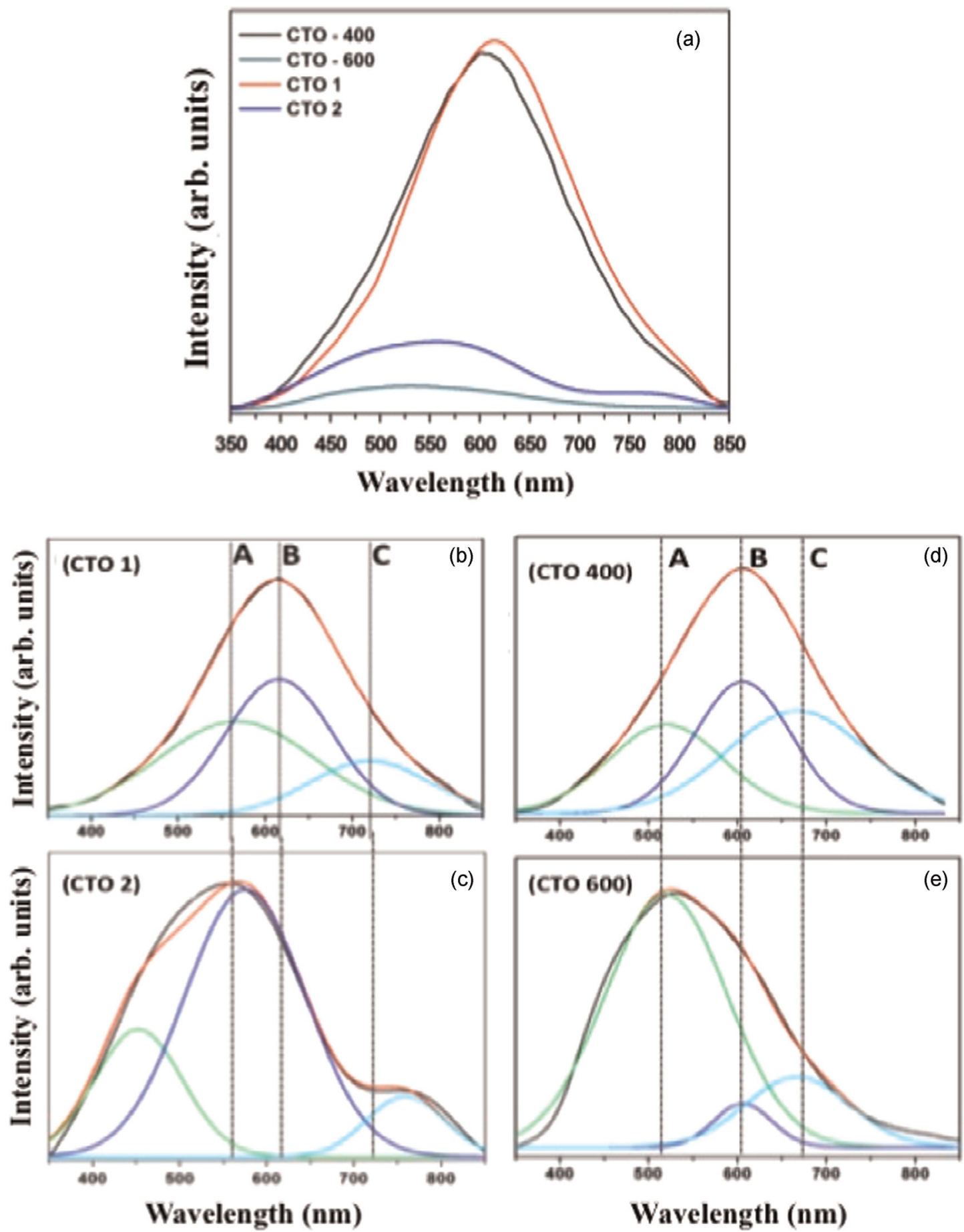

Fig. 6. PL spectra and PL spectra decomposition analyses of CTO 1, СTO 2, CTO 400 and CTO 600 powders.

Each color represents a different type of electronic transition and is linked to a specific structural arrangement regarding defects in the structure. These defects usually can be generated by oxygen vacancies for random common "perovskites" and/or disorder coupled to the "tilt" of a $\left[\mathrm{TiO}_{6}\right]-\left[\mathrm{TiO}_{6}\right]$ complex cluster $[42,43]$. Thus, the formation of an octahedron provides a degeneracy loss of titanium $3 \mathrm{~d}$ states in $\mathrm{e}_{\mathrm{g}}$ and $\mathrm{t}_{2 \mathrm{~g}}$ due to the Jahn-Teller effect. Local distortions and random vacancies can lead to hybridization of these states. Therefore, internal states will form in the band gap (see Fig. 5 (B) and (C)) as demonstrated previously for CTO compounds by UV-vis absorptions $[5,44]$.
To verify the effect of different precursors on the local structural order of the powders, XANES spectra were taken. As a reference, the СТО compound was synthesized by the Pechini method and heat-treated at $600{ }^{\circ} \mathrm{C}$ (CTO 600) and $400{ }^{\circ} \mathrm{C}$ (CTO 400 ), respectively. The use of these reference compounds illustrates that all $\mathrm{Ti}$ atoms are coordinated by six oxygen atoms in a completely regular octahedron $\left[\mathrm{TiO}_{6}\right]-\left[\mathrm{TiO}_{6}\right]$ for the samples heattreated at $600{ }^{\circ} \mathrm{C}$ (CTO 600) and coordinated by distorted $\left[\mathrm{TiO}_{6}\right]-\left[\mathrm{TiO}_{5}\right]$ complex clusters for the samples heat-treated at $400{ }^{\circ} \mathrm{C}$ (CTO 400). Fig. 8a and b shows normalized XANES spectra of CTO 1 and CTO 2 powders and the reference compounds (CTO 
Table 2

Evolution of PL emission for CTO powders

\begin{tabular}{lllllll}
\hline Sample & $\begin{array}{l}\text { Center } \\
\text { peak A }\end{array}$ & $\begin{array}{l}\text { Peak } \\
\text { area A } \\
(\%)\end{array}$ & $\begin{array}{l}\text { Center } \\
\text { peak B }\end{array}$ & $\begin{array}{l}\text { Peak } \\
\text { area B } \\
(\%)\end{array}$ & $\begin{array}{l}\text { Center } \\
\text { peak C }\end{array}$ & $\begin{array}{l}\text { Peak } \\
\text { area C } \\
(\%)\end{array}$ \\
\hline CTO 1 & 567 & 41 & 615 & 40 & 720 & 19 \\
CTO 2 & 459 & 14 & 565 & 78 & 771 & 8 \\
CTO 400 & 519 & 27 & 605 & 34 & 667 & 39 \\
CTO 600 & 519 & 76 & 605 & 6 & 667 & 18 \\
\hline
\end{tabular}

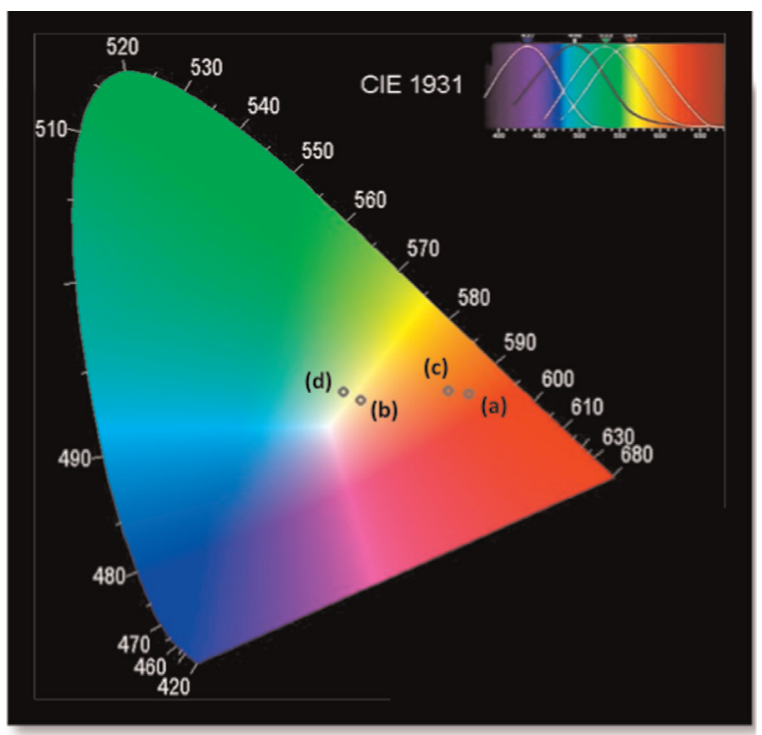

Fig. 7. The CIE chromaticity diagram for (a) CTO 1, (b) CTO 2 (c) CTO 400 and (d) CTO 600 powders.

400 and CTO 600) XANES spectra revealed differences in the preand post-edge regions (see Fig. 8a). In the post-edge region, a more ordered structure could be observed for the CTO 600 compound.

Fig. 8b shows a magnified view of the pre-edge region of powders studied in comparison with reference compounds. The intensity of the pre-edge peak (identified as *), is related to the $\left[\mathrm{TiO}_{5}\right]$ cluster concentration [10]. As expected, in the CTO 400 sample, the pre-edge peak revealed more intensity as compared to the other samples studied which occurs due to the co-existence of two environmental types of titanium atoms: a fivefold coordinated $\mathrm{TiO}_{5}$ (square-base pyramid) and a sixfold coordinated $\mathrm{TiO}_{6}$, (octahedron) before reaching the complete structural order. On the other hand, the CTO 600 sample shows less peak A intensity and consists of only $\left[\mathrm{TiO}_{6}\right]-\left[\mathrm{TiO}_{6}\right]$ (six-fold) complex clusters as reported previously $[5,10,30]$. An analysis of Peak A in Fig. 8b for CTO 1 powder shows more intensity as compared to the CTO 2 powder which indicates that the local Ti symmetry has a higher degree of distortion in the CTO 1 powder as compared to the CTO 2 powder.

The increase in the distortion could be caused by the coexistence of a complex-cluster-type $\left[\mathrm{TiO}_{5}\right]-\left[\mathrm{TiO}_{6}\right]$ complex cluster which is comparable to the CTO 400 sample. Interestingly, in the CTO 2 powder, the degree of disorder of the $\left[\mathrm{TiO}_{6}\right]$ cluster is lower than the degree of disorder in the CTO 1 powder but is larger than the CTO 600 sample.

These results shows that different materials employed as precursors (such as TTP and TC) produces CTO structures with a different local order around Ti atoms which is in good agreement with Raman and UV-vis results. Wavelength values for A and B emission peaks listed in Table 2 were analyzed. For the CTO
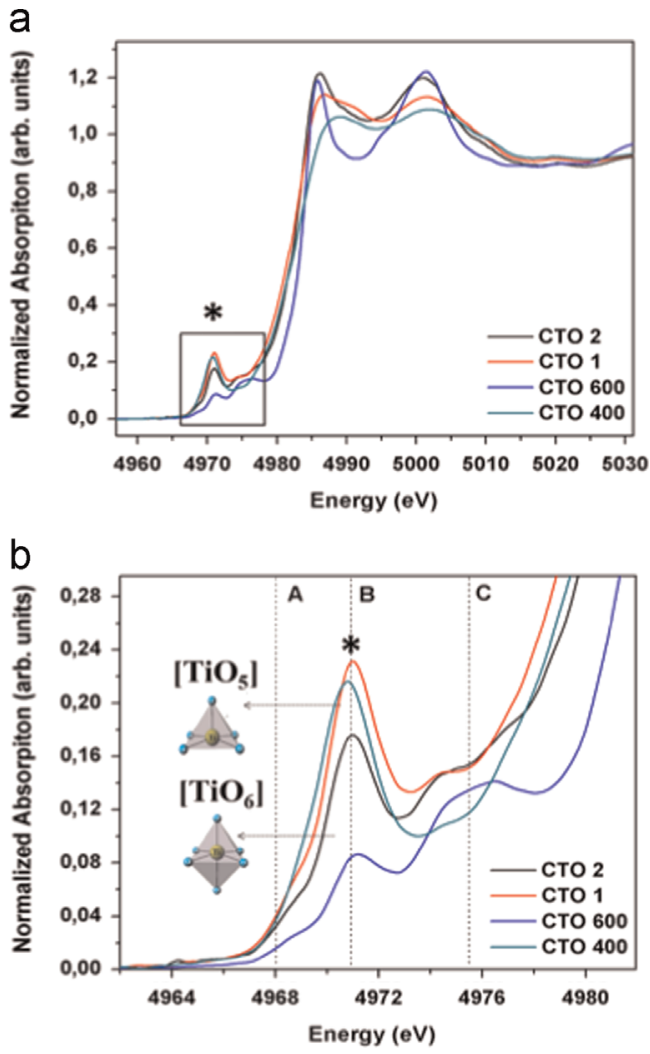

Fig. 8. (a) Normalized Ti K-edge XANES spectra and (b) the expanded view of the pre-edge region of normalized XANES for reference samples (CTO 400 and CTO 600) and CTO 1 and CTO 2 powders.

1 powder, these values can be associated with electron-hole recombination centers promoted by high energetic defects while the wavelength values and for the CTO 2 can be related to low energetic defects [11]. These results corroborate XRD, FT-Raman, UV-visible and XANES spectroscopy observations where the CTO 2 powder is more orderly in the long-, medium- and short-range. These results also indicate that the most important contribution in the substitution of the network formers is a shift to more energetic (shorter wavelength) components of visible light (shift to the left).

Structure defects of CTO powders can be due to oxygen vacancies $\left[\mathrm{TiO}_{5}\right]-\left[\mathrm{TiO}_{6}\right]$ in complex clusters in the CTO 1 powder and twists in bonding between the $\left[\mathrm{TiO}_{6}\right]-\left[\mathrm{TiO}_{6}\right]$ complex powder as observed by XANES spectroscopy [5]. Therefore, the use of different Ti precursors of the former network instead of $\mathrm{MAH}$ processing promotes a different intrinsic disorder in long-, shortand medium-range order in crystalline orthorhombic powders for the $\left[\mathrm{TiO}_{6}\right]$ cluster which is responsible for the PL property in CTO 1 and CTO 2 powders. Thus, we demonstrate that PL emission is sensitive to the organizational level of the system, and the use of PL spectroscopy is crucial as a very sensitive probe to analyze the degree of structural order-disorder at medium range in the ceramic materials lattice. Basically, the origin of PL emission in CTO powders is possible due to symmetry breaking in the lattice. In our band model (wide band model) before PL processing, defects facilitate an inhomogeneous charge distribution between $\left[\mathrm{TiO}_{6}\right]-\left[\mathrm{TiO}_{5}\right]^{\bullet}$ clusters or a pair of electrons $\left(\mathrm{e}^{\prime}\right)$-hole $\left(\mathrm{h}^{\bullet}\right)[3,11]$. These characteristics of the lattice promote the formation of new intermediary levels in the band gap. These structural changes can be related to polarization in different ranges that are, at the least, manifestations of possible quantum confinement which occurs at short- and intermediate-ranges independent of the particle size. 


\section{Conclusions}

In summary, these results show the versatility and advantages of microwave processing on CTO powders. Furthermore, it is possible to obtain these powders in a short time with low energy use. In addition, the use of different titanium precursors for the former network enhances the structural evolution of the material. The disorder coupled to the oxygen vacancies of $\left[\mathrm{TiO}_{5}\right]-\left[\mathrm{TiO}_{6}\right]$ in complex clusters in the CTO 1 powder and twists in bonding between $\left[\mathrm{TiO}_{6}\right]-\left[\mathrm{TiO}_{6}\right]$ clusters in the CTO 2 powder were mainly responsible for PL emission of a $\mathrm{CTO}_{\mathrm{MAH}}$ orthorhombic structure as a result of structural distortion. FE-SEM was used to follow the growth process from nanocrystalline-like building units to microcubes-shaped. A nucleation and crystal growth mechanism of micro-cube-shaped $\mathrm{CaTiO}_{3}$ is proposed to illustrate nanoparticle aggregation via $\mathrm{OH}$ groups adsorbed on the surfaces of selfassembly nanoplates.

\section{Acknowledgments}

The authors gratefully acknowledge the financial support of the Brazilian research funding agencies CAPES, FAPESP (2008/5787-1), INCTMN/CNPq (573636/2008-7) and FAPESP/CDMF (2013/072962). This work was partially developed at LNLS-Campinas, SP, Brazil.

\section{References}

[1] Z.H. Dong, T.N. Ye, Y. Zhao, J. Yu, F. Wang, L. Zhang, X. Wang, S. Guo, J. Mater. Chem. 21 (2011) 5978.

[2] M.L. Moreira, G.P. Mambrini, D.P. Volanti, E.R. Leite, M.O. Orlandi, P.S. Pizani, V.R. Mastelaro, C.O. Paiva-Santos, E. Longo, J.A. Varela, Chem. Mater. 20 (2008) 5381.

[3] F.V. Motta, A.T. de Figueiredo, V.M. Longo, V.R. Mastelaro, A.Z. Freitas, L. Gomes, N.D. Vieira Jr., E. Longo, J.A. Varela, J. Lumin. 129 (2009) 686.

[4] S.Y. Lee, M.C.C. Custodio, H.J. Lim, R.S. Feigelson, J.P. Maria, S. Trolier-McKinstry, J. Cryst. Growth 226 (2001) 247.

[5] M.L. Moreira, E.C. Paris, G.S. do Nascimento, V.M. Longo, J.R. Sambrano, V.R. Mastelaro, M. Bernardi, J. Andrés, J.A. Varela, E. Longo, Acta Mater. 57 (2009) 5174.

[6] S. Komarneni, R. Roy, Q.H. Li, Mater. Res. Bull. 27 (1992) 1393.

[7] C.H. Hsu, C.H. Lu, J. Mater. Chem. 21 (21) (2011) 2932.

[8] W. Sun, C. Li, J. Li, W. Liu, Mater. Chem. Phys. 97 (2006) 481.

[9] S. Komarneni, R.K. Rajha, H. Katsuki, Mater. Chem. Phys. 61 (1999) 50.

[10] J.C. Jan, K.P.K. Kumar, J.W. Chiou, H.M. Tsai, H.L. Shih, H.C. Hsueh, S.C. Ray, K. Asokan, W.F. Pong, M.H. Tsai, S.Y. Kuo, W.F. Hsieh, Appl. Phys. Lett. 81 (2003) 3311.

[11] F.M. Pontes, E. Longo, E.R. Leite, E.J.H. Lee, J.A. Varela, P.S. Pizani, C.E.M. Campos, F. Lanciotti, V.R. Mastelaro, C.D. Pinheiro, Mater. Chem. Phys, 77 (2003) 598.
[12] F. Farges, G.E. Brown, J.J. Rehr, Phys. Rev. B 56 (1997) 1809.

[13] S. de Lazaro, J. Milanez, A.T. de Figueiredo, V.M. Longo, Appl. Phys. Lett. 90 (2007) 111904.

[14] V.M. Longo, L.S. Cavalcante, R. Erlo, V.R. Mastelaro, A.T. de Figueiredo, J.R. Sambrano, S. de Lázaro, A.Z. Freitas, L. Gomes, N.D. Vieira Jr., J.A. Varela, E. Longo, Acta Mater. 56 (2008) 2191.

[15] B.L. Newalkar, S. Komarneni, H. Katsuki, Mater. Res. Bull. 36 (2001) 2347.

[16] G.J. Wilson, A.S. Matijasevich, D.R.G. Mitchell, J.C. Schulz, D.W. Geoffrey, Langmuir 22 (2006) 2016.

[17] K.J. Rao, M. Ganguli, P.A. Ramakrishnan, Chem. Mater. 11 (1999) 882.

[18] S. Said, P. Marchet, T. Merle-Mejean, J.P. Mercurio, Mater. Lett. 58 (2004) 1405.

[19] S. Bastide, D. Duphil, J.P. Borra, C. Levy-Clement, Adv. Mater. 18 (2006) 106.

[20] P. Podbrscek, G. Drazic, J.A. Paramo, Y.M. Strzhemechny, J. Macek, Z.C. Orel, CrystEngComm 16 (2010) 11481.

[21] D.P. Volanti, M.O. Orlandi, J. Andres, E. Longo, CrystEngComm 12 (2010) 1696.

[22] S. Mann, Biomineralization: Principles and Concepts in Bioinorganic Materials Chemistry, Oxford University Press, Oxford, 2001.

[23] J. Rieger, E. Hadicke, I. URau, D. Boeckh, Tenside Surfactants Deterg. 24 (1997) 430.

[24] O. Sohnel, J.W. Mullin, J. Cryst. Growth 60 (1982) 239.

[25] D. Schwahn, Y.R. Ma, H. Colfen, J. Phys. Chem. C 111 (2007) 3224.

[26] M.L. Moreira, V.M. Longo, W. Avansi Jr., M.M. Ferrer, J. Andres, V.R. Mastelaro, J.A. Varela, E. Longo, J. Phys. Chem. C 116 (2012) 24792.

[27] J.F. Banfield, S.A. Welch, H.Z. Zhang, T.T. Ebert, R.L. Penn, Science 289 (2000) 289751.

[28] F.M. Pontes, C.D. Pinheiro, E. Longo, E.R. Leite, S.R. de Lazaro, J.A. Varela, P.S. Pizani, T.M. Boschi, F. Lanciotti, Mater. Chem. Phys. 78 (2003) 227.

[29] S. de Lazaro, J. Milanez, A.T. de Figueiredo, V.M. Longo, V.R. Mastelaro, F.S. De Vicente, A.C. Hernandes, J.A. Varela, E. Longo, Appl. Phys. Lett. 90 (2007) 111904.

[30] J. Milanez, A.T. de Figueiredo, S. de Lazaro, V.M. Longo, R. Erlo, V.R. Mastelaro, R.W.A. Franco, E. Longo, J.A. Varela, J. Appl. Phys. 106 (2009) 043526.

[31] H. Zheng, I.M. Reaney, G. de Gyorgyfalva, R. Ubic, J. Yarwood, M.P. Seabra, V.M. Ferreira, J. Mater. Res. 19 (2004) 488.

[32] S. Qin, X. Wu, F. Seifert, A.I. Becerro, J. Chem. Soc. Dalton Trans. 19 (2002) 19 3751.

[33] T.M. Mazzo, M.L. Moreira, I.M. Pinatti, F.C. Picon, E.R. Leite, I.L.V. Rosa, J.A. Varela, L.A. Perazolli, E. Longo, Opt. Mater. 32 (2010) 990.

[34] U. Balachandran, N.G. Eror, Solid State Commun. 44 (1982) 815.

[35] S. Qin, X. Wu, F. Seifert, A.I. Becerro, J. Chem. Soc. Dalton Trans. 19 (2002) 3751.

[36] Y. Li, S. Qin, F. Seifert, J. Solid State Chem. 22 (2007) 180.

[37] H. Zheng, G.D.C.C. Gyorgyfalva, R. Quimby, H. Bagshaw, I.M. Reaney, J. Yarwood, J. Eur. Ceram. Soc. 23 (2003) 2653.

[38] C. Malibert, B. Dkhil, J.M. Kiat, D. Durand, J.F. Berar, A. SpasojevicdeBire, J. Phys. Condens. Matter 9 (1997) 7485.

[39] L.S. Cavalcante, V.S. Marques, J.C. Sczancoski, M.T. Escote, M.R. Joya, J.A. Varela, M.R.M.C. Santos, P.S. Pizani, E. Longo, Chem. Eng. J. 143 (2008) 299.

[40] D.L. Wood, J. Tauc, Phys. Rev. B 5 (1972) 3144.

[41] K.M.M.K. Prasad, S. Raheem, P. Vijjayalekshmi, C.K. Sastri, Talanta 43 (1996) 1187.

[42] S. Banerjee, D.I. Kim, R.D. Robinson, I.P. Herman, Y.B. Mao, S.S. Wong, Appl. Phys. Lett. 89 (2006) 223130.

[43] V.M. Longo, A.T. de Figueiredo, S. de Lazaro, M.F. Gurgel, M.G.S. Costa, C.O. Paiva-Santos, J.A. Varela, E. Longo, V.R. Mastelaro, F.S. de Vicente, A.C. Hernandes, R.W.A. Franco, J. Appl. Phys. 104 (2008) 023515.

[44] M. Hiroki, H. Tamae, O. Tomohisa, M. Koji, J. Mitsuru, K. Hisao, Fish. Sci. 4 (2008) 418 . 